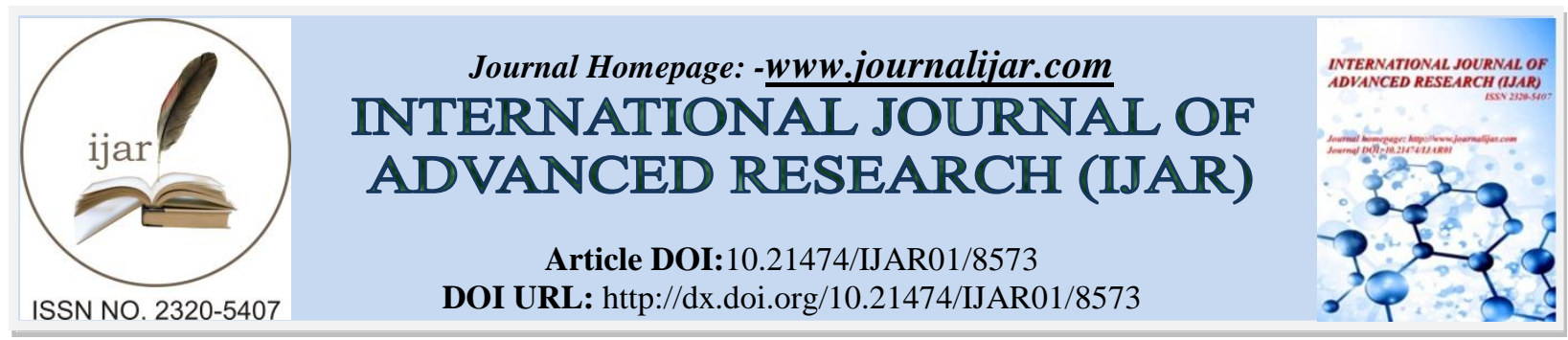

RESEARCH ARTICLE

\title{
THE EFFECT OF FINANCIAL PERFORMANCE OF SOPPENG REGENCY GOVERNMENT ON COMMUNITY PROSPERITY LEVEL.
}

Darmawati Aras ${ }^{1}$, Abdu Hamid Habbe ${ }^{2}$ and Arifuddin ${ }^{2}$.

1. Master of Accounting Depatrtment, Faculty of Economics and Bussiness, HasanuddinUniversity.

2. Faculty of Economics and Bussiness,Hasanuddin University.

\section{Manuscript Info}

Manuscript History

Final Accepted: 16 January 2019

Published: February 2019

\section{Key words:-}

Independence ratio, effectiveness ratio, budget absorption ratio, compatibility ratio, community prosperity.
Received: 14 December 2018

\begin{abstract}
The research aimed to perceive the regional autonomy implementation at Soppeng Regency by investigating: 1) whether the financial independence level through the expenditure compatibility and budget absorption could improve the community prosperity; 2) whether the effectiveness level of the Regional Original Revenue (ROR) through the expenditure compatibility and budget absorption could improve the community prosperity.

The research used the quantitative approach the time series as the secondary data for ten years (from 2007 to 2016). Data were analysed using the path analysis to find out the effect of the independent variable on the dependent variabel with the significance level of 5\%. The research data were analysed using Eviews.

The research result indicates that the financial independence throught the expenditure compatibility and budget absorption has the positive and significant relationship with IPM, however, the effectiveness of ROR through the expenditure compatibility and budget absorption has the positive and insignificant relationship with IPM. This also indicates that the regional autonomy implementation at Soppeng Regency has not fully fulfilled the mandate of autonomy acts which is expected to be able to optimize the economic potentials in order to improve the community prosperity.
\end{abstract}

Copy Right, IJAR, 2019,. All rights reserved.

\section{Introduction:-}

Indonesia changed their policy into regional autonomy when Act 22 of 1999 changed to Act 32 of 2004, later changed to Act 23 of 2014 on Regional Government, and Act 25 on 1999 later changed to Act 33 of 2004 on Monetary Balancing between Central and Regional Government. With the enactment of regional autonomy, regional developments need to be carefully planned. This can be achieved by a careful development budgeting planning and supported with a well-managed regional income. Regional autonomy and fiscal decentralization are enacted by considering that regional governments are more aware with the needs and standards of service of their peoples. Therefore, regional autonomy is hoped to improve people' welfare through the improvements of local economy as reflected in ProdukDomestik Regional Bruto (PDRB).

One of few measurement means that could analyze governments' performance in managing their regional budget is by employing monetary ratio analysis on the specified and implemented APBD (Halim, 2007:231). Some monetary

Corresponding Author:-Darmawati Aras.

Address:-Master of Accounting Depatrtment, Faculty of Economics and Bussiness, 
ratios used to measure the accountability of regional government are independent ratio, regional income effectiveness ratio, regional budget efficiency ratio, and expenditure conformity ratio. In addition, United Nations Development Program (UNDP) measure people' welfare comprehensively by calculating income per capita, life expectancy, educational level which are constructed into Human Development Index.

Empiric studies on regional monetary performance is common in Indonesia, some are intended to evaluate the monetary performance of regional government. This indicated a huge concern on the quality of regional institution performance, especially in their service.

Analysis of Hendarmin (2012) in Kalimantan Barat on the influence of capital expenditure and private investment on economic growth, work opportunities, people welfare concluded that generally welfare could be improved by improving capital expenditure, that broaden work opportunities and eventually improving people welfare.

Study conducted by Swandewi (2014) proved that regional monetary independency indirectly have a significant effect on people welfare through budget conformity. Regional government independency ratio reflected regional autonomy capability that is measured by the amount of PendapatanAsli Daerah (PAD) compared to total regional income. This result is problematic; independent regency could improve their capital expenditure in exchange for public service. Ardhini (2012) proved that regional income effectiveness ratio positively influencing capital expenditure allocation addressed to public service. Therefore, if regional monetary tends to be effective, it would affect capital expenditure spend on public service. Study conducted by Wahyudi and Rejekiningsih (2013) found that government spending on healthcare and education affect economic growth and poverty. However, Vegirawati (2012) found that direct spending could not predict Human Development Index.

Mirza (2012) proved that capital expenditure could improve Human Development Index. Capital expenditure conformity on APBD realization showed that government is concerned with regional development. Regional government monetary performance is hoped to be more concerned with people needs.

From the statement above, the researcher is interested to explore how monetary performance of KabupatenSoppeng affects people welfare. This study is intended to measure the achievement level of KabupatenSoppeng government in fulfilling the main objective of regional autonomy that is to ensure the wellbeing of people through an effective and efficient APBD management.

\section{Research Methodology:- \\ Research Planning}

This study employed quantitative approach. This study is a hypothesis testing, intended to analyze the correlation between regional monetary performance and people welfare. This study is a case study. During observation, a timeseries secondary data was employed.

\section{Location and Time}

This study was conducted in KabupatenSoppeng, specifically at Regional Government of KabupatenSoppeng and focused to Monetary Report. Study time was scheduled based on situation and condition of study location.

\section{Population and Sample}

The population of this study is regional monetary report audited by BPK and it was enacted as PerdaKabupatenSoppeng. Monetary report used is APBD realization report of 2007-2016. HDI data is also taken from similar timespan.

\section{Data Source and Type}

Quantitative data is used in this research. The source of data is secondary data collected from Accountancy Board of Regional Equipment and Finance Office of KabupatenSoppeng. Data on people welfare is collected from BPS KabupatenSoppeng and Regional Development Planning Board (Bappeda) KabupatenSoppeng as cited in "Soppeng in Angka" book.

\section{Data Collection Method}

Methods employed in this research are documentation and literature. Data collected are thos related with regional monetary performance ratio calculation and welfare available in BPS office and Bappeda. 


\section{Research Variables}

This study is comprised of two independent variables, two intervening variables, and one dependent variable. Monetary performance is reflected on independent variables that are Monetary Independency Ratio, PAD Effectiveness Ratio. Intervening variables are Budget Absorption Ratio, Expenditure Conformity Ratio. Dependent variables consisted of People Welfare reflected on IPM (Income per Capita, Education and Healthcare Indicator)

\section{Data Analysis Technique:-}

Path analysis is employed in this study, with regression equation as follows:

First Analysis, to test the influence of independent variables $\left(\mathrm{X}_{1}\right.$ and $\left.\mathrm{X}_{2}\right)$ on intervening variables $\left(\mathrm{X}_{3}\right.$ and $\left.\mathrm{X}_{4}\right)$, illustrated as:

Figure 4.2

Effect of Independent Variables on Intervening Variables

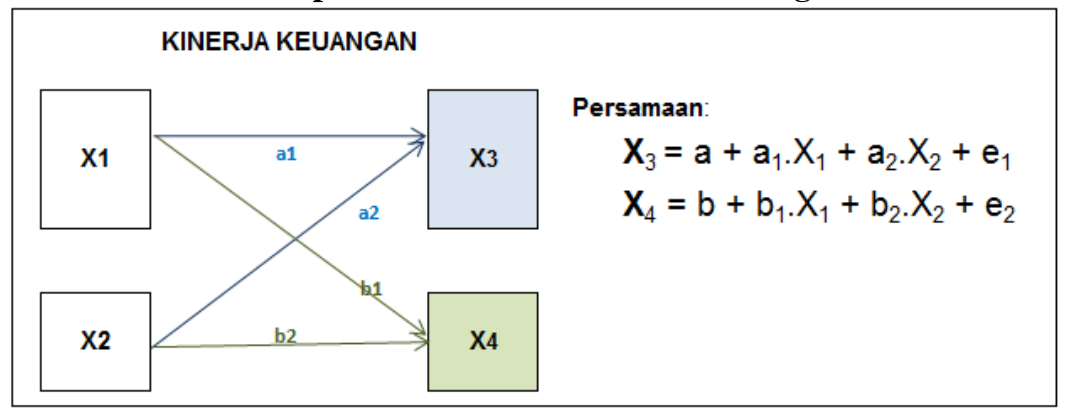

\section{Information:}
$\mathrm{a}=$ the constant effect $\mathrm{X}_{1}$ and $\mathrm{X}_{2}$ on $\mathrm{X}_{3}$
$\mathrm{a}_{1}=$ the effect $\mathrm{X}_{1}$ on $\mathrm{X}_{3}$
$\mathrm{a}_{2}=$ the effect $\mathrm{X}_{2}$ on $\mathrm{X}_{3}$
$\mathrm{b}=$ the effect constant $\mathrm{X}_{1}$ and $\mathrm{X}_{2}$ on $\mathrm{X}_{4}$
$\mathrm{b}_{1}=$ the effect $\mathrm{X}_{1}$ on $\mathrm{X}_{4}$
$\mathrm{b}_{2}=$ the effect $\mathrm{X}_{2}$ on $\mathrm{X}_{4}$
$\mathrm{e}_{1}=$ standard error of effect of $\mathrm{X}_{1}$ and $\mathrm{X}_{2}$ on $\mathrm{X}_{3}$
$\mathrm{e}_{2}=$ standard error of effect of $\mathrm{X}_{1}$ and $\mathrm{X}_{2}$ on $\mathrm{X}_{4}$

Second Analysis, to understand the influence of intervening variables $\left(\mathrm{X}_{3}\right.$ and $\left.\mathrm{X}_{4}\right)$ on dependent variable $(\mathrm{Y})$, illustrated as:

Gambar 4.3:-PengaruhVariabel Intervening terhadapVariabelDependen

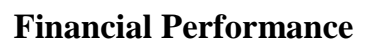

\section{Community Prosperity}

\section{$\mathrm{k} 1$}

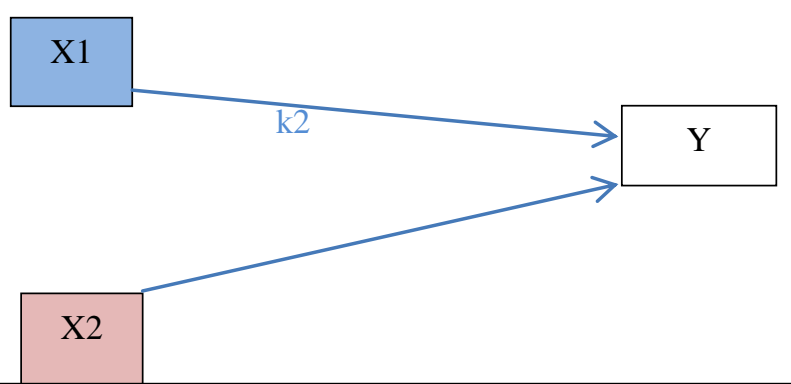

\section{Equation:}

$\mathrm{Y}_{(\mathrm{n}+1,2,3,4)}=\mathrm{k}+\mathrm{k}_{1} \cdot \mathrm{X}_{3(\mathrm{tn})}+\mathrm{k}_{2} \cdot \mathrm{X}_{4(\mathrm{n}))}+\mathrm{e}_{3}$ 


\section{Information:}

$\mathrm{k}=$ the constant effect $\mathrm{X}_{3}$ and $\mathrm{X}_{4}$ on $\mathrm{Y}_{1}$

$\mathrm{k}_{1}=$ the effect $\mathrm{X}_{3}$ on $\mathrm{Y}$

$\mathrm{k}_{2}=$ the effect $\mathrm{X}_{4}$ on $\mathrm{Y}$

$\mathrm{e}_{3}=$ standard error of effect of $\mathrm{X}_{3}$ and $\mathrm{X}_{4}$ on $\mathrm{Y}_{1}$

\section{Result:-}

Data Description

Independent variables consisted of Independency Ratio and Effectiveness Ratio as collected from 2007-2016 as follows:

\section{Independence Ratio \& Effectiveness Ratio}

\begin{tabular}{|c|c|c|c|}
\hline \multirow{2}{*}{ No. } & \multirow{2}{*}{ Year } & \multicolumn{2}{|c|}{ Independent Variables } \\
\cline { 3 - 4 } & & Independence Ratio & Effectiveness Ratio \\
\cline { 3 - 4 } & 2007 & 4,25 & $\mathbf{X 2}$ \\
\hline 1 & 2008 & 4,09 & 106,82 \\
\hline 2 & 2009 & 3,62 & 130,12 \\
\hline 3 & 2010 & 3,39 & 89,28 \\
\hline 4 & 2011 & 3,73 & 80,95 \\
\hline 5 & 2012 & 4,24 & 112,34 \\
\hline 6 & 2013 & 5,56 & 107,85 \\
\hline 7 & 2014 & 7,71 & 116,69 \\
\hline 8 & 2015 & 7,12 & 120,13 \\
\hline 9 & 2016 & 8,10 & 117,96 \\
\hline 10 & & 1.593 .481 & 109,41 \\
\hline \multicolumn{2}{c|}{} & 1.445 .666 & 4.684 .104 \\
\hline \multicolumn{2}{c|}{ Median } & 2.091 .948 & 4.708 .331 \\
\hline \multicolumn{2}{c|}{ Maximum } & 1.219 .830 & 4.868 .449 \\
\hline \multicolumn{2}{c|}{ Std. Dev. } & 0.331565 & 4.393 .771 \\
\hline \multicolumn{2}{c}{ Probability } & 0.485543 & 0.141824 \\
\hline
\end{tabular}

Source: Data Processed

Intervening variables consisted of Expenditure Conformity Ratio and Budget Absorption Ratio as collected from 2007-2016 as follows:

Expenditure Conformity Ratio \& Budget Absorption Ratio

\begin{tabular}{|c|c|c|c|}
\hline \multirow{2}{*}{ No. } & \multirow{2}{*}{ Years } & \multicolumn{2}{|c|}{ Intervening variables } \\
\cline { 3 - 4 } & & Expenditure Conformity Ratio & Budget Absorption Ratio \\
\cline { 3 - 4 } & 2007 & 62,16 & $\mathbf{X 4}$ \\
\hline 1 & 2008 & 62,32 & 90,57 \\
\hline 2 & 2009 & 58,47 & 91,01 \\
\hline 3 & 2010 & 48,51 & 98,46 \\
\hline 4 & 2011 & 53,16 & 96,59 \\
\hline 5 & 2012 & 40,48 & 94,32 \\
\hline 6 & 2013 & 43,77 & 92,89 \\
\hline 7 & 2014 & 51,11 & 92,64 \\
\hline 8 & 2015 & 56,43 & 94,88 \\
\hline 9 & 2016 & 58,82 & 92,66 \\
\hline 10 & & 49.99073 & 90,88 \\
\hline \multicolumn{2}{|c|}{ Mean } & 49.40616 & 3.970 .679 \\
\hline \multicolumn{2}{|c|}{ Median } & & 4.003 .139 \\
\hline
\end{tabular}




\begin{tabular}{|c|c|c|}
\hline Maximum & 83.03544 & 4.132 .275 \\
\hline Minimum & 28.87895 & 3.700 .914 \\
\hline Std. Dev. & 16.21263 & 0.146964 \\
\hline Probability & 0.761934 & -0.623318 \\
\hline
\end{tabular}

Source: Data Processed

Dependent variable consisted of Human Development Index (HDI) in Soppeng Regency as collected from 20072016 as follows:

Human Development Index

\begin{tabular}{|c|c|c|}
\hline \multirow{2}{*}{ No. Year } & Dependent Variables \\
\cline { 3 - 3 } & & HDI \\
\cline { 2 - 3 } & & 62,45 \\
\hline 1 & 2007 & 62,92 \\
\hline 2 & 2008 & 63,10 \\
\hline 3 & 2009 & 63,51 \\
\hline 4 & 2010 & 63,80 \\
\hline 5 & 2011 & 64,05 \\
\hline 6 & 2012 & 64,43 \\
\hline 7 & 2013 & 64,74 \\
\hline 8 & 2014 & 65,33 \\
\hline 9 & 2015 & 65,95 \\
\hline 10 & 2016 & 4.159 .187 \\
\hline \multicolumn{2}{|c|}{} & 4.157 .709 \\
\hline \multicolumn{2}{|c|}{ Mean } & 4.188 .897 \\
\hline \multicolumn{2}{|c|}{ Maximum } & 4.134 .366 \\
\hline \multicolumn{2}{|c|}{ Minimum } & 0.017194 \\
\hline \multicolumn{2}{|c|}{ Probability } & 0.279716 \\
\hline
\end{tabular}

Source: BPS of Soppeng Regency (2018)

\section{Result of Influence of Independent Variables on Intervening Variables}

The result of statistic test on Independency Ratio and Effectiveness Ratio variables on Expenditure Conformity Ratio illustrated below:

The Effect $X_{1}$ and $X_{2}$ on

Date: 08/29/18 Time: 05:07

Sample: 20072016

Included observations: 10

Family: Normal

Link: Identity

Dispersion computed using Pearson Chi-Square

Convergence achieved after 1 iteration

Coefficient covariance computed using observed Hessian

\begin{tabular}{|c|r|r|r|r|}
\hline & & & & \\
\hline Variable & Coefficient & Std. Error & z-Statistic & Prob. \\
\hline X1 & & & & \\
\hline X2 & 2.188446 & 0.987685 & 2.215732 & 0.0267 \\
\hline $\mathrm{C}$ & 71.57516 & 21.04659 & 3.400796 & 0.9369 \\
\hline & & & & 0.0007 \\
\hline Mean dependent var & 95.91730 & S.D. dependent var & & 25.82611 \\
\hline
\end{tabular}




\begin{tabular}{|l|r|l|r|}
\hline Sum squared resid & 3469.020 & Log likelihood & -43.71797 \\
\hline Akaike info criterion & 9.343594 & Schwarz criterion & 9.434370 \\
\hline Hannan-Quinn criter. & 9.244014 & Deviance & 3469.020 \\
\hline Deviance statistic & 495.5742 & Restr. deviance & 6002.891 \\
\hline LR statistic & 5.113001 & Prob(LR statistic) & 0.077576 \\
\hline Pearson SSR & 3469.020 & Pearson statistic & 495.5742 \\
\hline Dispersion & 495.5742 & & \\
\hline & & & \\
\hline
\end{tabular}

Source: The Result of Statistical Test (Eviews Version 7)

The figure showed the result of equation $\mathrm{X} 3=\mathrm{a}+\mathrm{a} 1 . \mathrm{X} 1+\mathrm{a} 2 \mathrm{X} 2+\mathrm{e} 1$ with regression equation:

$\mathrm{X} 3=\mathbf{7 1 . 5 7 5 1 6}+\mathbf{2 . 1 8 4 4 6}+\mathbf{0 . 0 1 3 7 6 5}+\mathrm{e}$

Probability value of each variable $\mathrm{X} 1$ on variable $\mathrm{X} 3$ showed a significant value of $\leq 5 \%$ with probability value of 0,0267 , whilst variable $\mathrm{X} 2$ on variable $\mathrm{X} 3$ showed an insignificant value of $\geq 5 \%$ with probability value of 0,9369 , exceeding the required probability value. This indicated that Independency Ratio significantly influences Expenditure Conformity Ratio, in contrast with Effectiveness Ratio which insignificantly influences Expenditure Conformity Ratio. This might be caused by the fluctuating tendency of Effectiveness Ratio compared to the linier tendency of Expenditure Conformity Ratio. In addition, the population which only limited to one government body with 10 years data could influence the significant value of each relationship.

The result of statistic test on Independency Ratio and Effectiveness Ratio variables on Budget Absorption Ratio illustrated below:

The Effect $X_{1}$ and $X_{2}$ on $X_{4}$

Dependent Variable: $\mathrm{X} 4$

Method: Generalized Linear Model (Quadratic Hill Climbing)

Date: 08/29/18 Time: 05:08

Sample: 20072016

Included observations: 10

Family: Normal

Link: Identity

Dispersion computed using Pearson Chi-Square

Convergence achieved after 1 iteration

Coefficient covariance computed using observed Hessian

\begin{tabular}{|c|c|c|c|c|}
\hline Variable & Coefficient & Std. Error & z-Statistic & Prob. \\
\hline $\mathrm{X} 1$ & 2.763606 & 1.323430 & 2.088215 & 0.0368 \\
\hline $\mathrm{X} 2$ & -0.251348 & 0.233137 & -1.078117 & 0.2810 \\
\hline $\mathrm{C}$ & 60.08211 & 28.20097 & 2.130498 & 0.0331 \\
\hline Mean dependent var & 61.89530 & S.D. dependent var & & 34.31902 \\
\hline Sum squared resid & 6228.326 & Log likelihood & & -46.64415 \\
\hline Akaike info criterion & 9.928830 & Schwarz criterion & & 10.01961 \\
\hline Hannan-Quinn criter. & 9.829249 & Deviance & & 6228.326 \\
\hline Deviance statistic & 889.7609 & Restr. Deviance & & 10600.15 \\
\hline LR statistic & 4.913487 & Prob(LR statistic) & & 0.085714 \\
\hline Pearson SSR & 6228.326 & Pearson statistic & & 889.7609 \\
\hline Dispersion & 889.7609 & & & \\
\hline
\end{tabular}

Source: The Result of Statistical Test (Eviews Version 7)

The figure showed the result of equation $\mathrm{X} 4=\mathrm{b}+\mathrm{b} 1 . \mathrm{X} 1+\mathrm{b} 2 \mathrm{X} 2+\mathrm{e} 1$ with regression equation: 


\section{$\mathrm{X} 4=60.08211+2.763606-0.251348+\mathrm{e}$}

Probability value of each variable X1 and X2 on variable X4 with significance level of $5 \%(0,05)$ showed that X1 significant on 0,0368 with z-Statistic value of 2.088215, shills X2 is insignificant on 0,2810 with z-Statistic value of $-1,07817$. From the equation above, it can be concluded that for every $1 \%$ increase of Independency Ratio, Budget Absorption Ratio will increase 2,76\% and Expenditure Conformity Ratio will be set on constant level of 60,08\% without the influence of either Independency Ratio or Effectiveness Ratio.

\section{Result of Influence of Intervening Variables on Dependent Variable}

Relationship between Expenditure Conformity and Budget Absorption Ratios on IPM is significant on Y1(tn+1) test illustrated as:

\section{The Effect $\mathrm{X}_{\mathbf{3}}$ and $\mathrm{X}_{4}$ on $\mathrm{Y}$}

\begin{tabular}{|c|c|c|c|c|}
\hline \multicolumn{3}{|l|}{ Dependent Variable: Y } & & \\
\hline \multicolumn{5}{|c|}{ Method: Generalized Linear Model (Quadratic Hill Climbing) } \\
\hline \multicolumn{5}{|c|}{ Date: $08 / 29 / 18$ Time: $05: 10$} \\
\hline \multicolumn{5}{|c|}{ Sample: 20072016} \\
\hline \multicolumn{5}{|c|}{ Included observations: 10} \\
\hline \multicolumn{5}{|c|}{ Family: Normal } \\
\hline \multicolumn{5}{|l|}{ Link: Identity } \\
\hline \multicolumn{5}{|c|}{ Dispersion computed using Pearson Chi-Square } \\
\hline \multicolumn{5}{|c|}{ Convergence achieved after 1 iteration } \\
\hline \multicolumn{5}{|c|}{ Coefficient covariance computed using observed Hessian } \\
\hline Variable & Coefficient & Std. Error & z-Statistic & Prob. \\
\hline $\mathrm{X} 3$ & 0.035291 & 0.017337 & 2.035604 & 0.0418 \\
\hline $\mathrm{X} 4$ & 0.028880 & 0.013047 & 2.213585 & 0.0269 \\
\hline $\mathrm{C}$ & 59.29215 & 1.945923 & 30.46995 & 0.0000 \\
\hline Mean dependent var & 64.46470 & S.D. dependent var & & 1.755487 \\
\hline Sum squared resid & 12.56697 & Log likelihood & & -15.61519 \\
\hline Akaike info criterion & 3.723039 & Schwarz criterion & & 3.813814 \\
\hline Hannan-Quinn criter. & 3.623458 & Deviance & & 12.56697 \\
\hline Deviance statistic & 1.795281 & Restr. Deviance & & 27.73563 \\
\hline LR statistic & 8.449185 & $\begin{array}{l}\operatorname{Prob}(\text { LR statistic) } \\
\end{array}$ & & 0.014631 \\
\hline Pearson SSR & 12.56697 & Pearson statistic & & 1.795281 \\
\hline Dispersion & 1.795281 & & & \\
\hline
\end{tabular}

Source: The Result of Statistical Test (Eviews Version 7)

The illustration showed the result of statistic test with equation $\mathrm{Y} 1=\mathrm{k}+\mathrm{k} 1 . \mathrm{X} 3+\mathrm{k} 2 . \mathrm{X} 3+\mathrm{e}$ with regression equation:

$$
\mathrm{Y} 1(\mathrm{tn}+1)=59.29115+0.035291 \mathrm{X} 3=0.028880 \mathrm{X} 4+\mathrm{e}
$$

From the equation, it can be explained that for every $1 \%$ increase of Expenditure Conformity Ratio, HDI will increase $0.0289 \%$. HDI will be set on 59,29\% without the influence of Expenditure Conformity Ratio and Budget Absorption Ratio.

Probability values of each variable $\mathrm{X} 3$ and $\mathrm{X} 4$ on variable $\mathrm{Y}$ with on significance level of 5\% $(0,05)$ showed that $\mathrm{X} 3$ gave positive and significant influence on $\mathrm{Y}$ with 0,0418 with t-Statistic value of 2.035604. Similar result on X5 showed significant influence on $\mathrm{Y}$ with probability value of 0.0269 with t-Staistic 2.213585 .

\section{Discussion:-}

The Influence of Independency Ratio through Expenditure Conformity on People Welfare

This study found that correlation coefficient of Independency Ratio (X1) through Expenditure Conformity Ratio (X3) on HDI (Y) showed positive and significant influence, meaning that $\mathrm{H} 1$ is accepted. The result strengthens 
Mirza's (2012) findings that economic growth has positive and significant impact on Human Development Index (HDI) of Government of Jawa Tengah. Moreover, Swandesi (2014) proved that regional monetary independency indirectly have positive and significant influence on people welfare through expenditure conformity in Bali. However, this study differs with study conducted in Sumatera Selatan by Vegirawati (2012) that found that expenditure could not predict Human Development Index.

\section{The Influence of Independency Ratio through Budget Absorption on People Welfare}

Correlation coefficient of Independency Ratio (X1) through Budget Absorption Ratio (X4) on HDI (Y) showed positive and significant influence, meaning that H2 is accepted. The result strengthens Mirza's (2012) findings that economic growth has positive and significant impact on Human Development Index (HDI) in Jawa Tengah. However, the findings are different compared to Vegirawati (2012) who found that expenditure could not predict Human Development Index.

\section{The Influence of PAD Effectiveness through Expenditure Conformity on People Welfare}

Correlation coefficient of Effectiveness Ratio (X2) through Expenditure Conformity (X3) on HDI (Y) showed positive but insignificant influence, meaning that $\mathrm{H} 3$ is rejected. The result generally showed that PAD effectiveness through expenditure conformity could not increase people welfare. Regional capability to optimize regional monetary potency is low therefore PAD targets set on APBD are still far from realization. Therefore, even if PAD realization peaked at $100 \%$ or more, it did not show the real performance. As conducted by Saputra (2014) in Sumatera Barat, PAD realization effectiveness tends to improve annually, yet it could not reduce the financial transfer from central government. This showed that regional monetary performance as measured by PAD realization effectiveness did not illustrate regional capabilities in managing their economic activity sources. Ideally, PAD targets draw the real potential and therefore PAD realization could picture the ongoing economic activities.

\section{The Influence of PAD Effectiveness through Budget Absorption on People Welfare}

Correlation coefficient of Effectiveness Ratio through Budget Absorption Ratio on HDI showed positive but insignificant influence, meaning that $\mathrm{H} 4$ is rejected. The result showed that regional monetary independency through budget absorption could improve people welfare. This discovery is against what Adrhini and Handayani (2012) found that PAD Effectiveness Ratio in regional monetary management had positive and significant influence on capital expenditure allocated to people welfare. Therefore, if regional monetary effectiveness tends to be effective, it could influence the amount of capital expenditure spend on people welfare. Mirza (2012) proved that capital expenditure enacted by government had positive and significant influence on HDI.

\section{Conclusion And Suggestions:-}

Monetary performance of a particular year significantly contributes to people welfare of the following year:

1. Monetary Independency Ratio through Expenditure Conformity Ratio could improve people welfare in KabupatenSoppeng in every indicator.

2. Independency Ratio through Budget Absorption Ratio could improve people welfare in KabupatenSoppeng in every indicator.

3. Effectiveness Ratio through Conformity Ratio and Budget Absorption Ratio could not improve people welfare in KabupatenSoppeng in every indicator.

4. Effectiveness Ratio through Conformity Ratio and Budget absorption Ratio could not improve people welfare in KabupatenSoppeng in every indicator.

It is suggested to future researchers interested with this topic to increase data population. This could be done by adding research locations. This could also act as comparison between regions.

\section{Acknowledgments:-}

For the completion of this research, the researcher would thank all parties involved in this study: Prof. Dr. Dwia Aries Tina Pulubuhu, MA, rector of UniversitasHasanuddin for the chance given to study in Accountancy Program at Faculty of Economy and Business, National Government Internal Auditor and ADB that provide the researcher with scholarship. Prof. Dr. Abd.RahmanKadir, SE., M.Si dean of Faculty of Economy and Business, Dr. R.A. Damayanti, SE., AK.,M.Soc.,Sc.,CA head of Accountancy Program for the chance given to study here, Dr. Abdul Hamid Habbe, SE.,M.Si as First Consultant and Dr. Arifuddin, SE.,Ak.,M.Si.,CA as Second Consultant for their guidance and knowledge in completing this thesis. 


\section{Daftar Pustaka:-}

1. Ardhini dan Sri Handayani, 2012. Pengaruh Rasio Keuangan Daerah Terhadap Belanja Modal untuk Pelayanan Publik dalam Perspektif Teori Keagenan (Studi pada Kabupaten dan Kota Di Jawa Tengah). Tidak dipublikasikan.

2. Arsyad, Lincolin. 2010. Ekonomi Pembangunan. CetakanPertama. Yogyakarta: UPP STIM YKPN.

3. Astri, M., Nikensari, S.I., danKuncara, H. 2013.PengaruhPengeluaranPemerintah Daerah padaSektorPendidikandanKesehatanTerhadapIndeks Pembangunan Manusia.JurnalPendidikanEkonomi Dan Bisnis. Vol. 1 No. 1.

4. Batafor, GregoriusGehi. 2011. EvaluasiKinerjaKeuangandan Tingkat KesejahteraanMasyarakatKabupatenLembata - Provinsi NTT.Tesis Program PascaSarjana.Denpasar.

5. Budiarto, Arif. 2007. Jasa-jasa Audit.Jakarta :SalembaEmpat.

6. Devas, Nick, dkk. 1989. KeuanganPemerintah Daerah di Indonesia. Jakarta: UI Press.

7. Fahmi, Irham. 2010. ManajemenKinerja: TeoridanAplikasi. Bandung: Alfabeta.

8. Fahmi, Irham. 2011. AnalisisKinerjaKeuangan. CetakanKesatu. Bandung: Alfabeta.

9. Halim, Abdul. 2007. AkuntansiSektorPublik: AkuntansiKeuangan Daerah. Jakarta: SalembaEmpat.

10. Hasanah, E.U., danSunyoto, D. 2012. PengantarIlmuEkonomiMakro. CetakanPertama. Yogyakarta: CAPS.

11. Hendarmin. 2012. PengaruhBelanja Modal Pemerintah Daerah danInvestasiSwastaterhadapPertumbuhanEkonomi, KesempatanKerjadanKesejahteraanMasyarakat di Kabupaten/Kota Provinsi Kalimantan Barat. JurnalEksos. Vol. 8 No. 3.

12. Jhingan, M.L. 1979. Ekonomi Pembangunan danPerencanaan.Penerjemah: D. Guritno. CetakanKeempatBelas. Jakarta: RajaGrafindoPersada.

13. Mahmudi. 2006. AnalisisLaporanKeuanganPemerintah Daerah. EdisiKedua. CetakanPertama. Yogyakarta: UPP STIM YKPN.

14. Mahmudi. 2009. ManajemenKeuangan Daerah. Jakarta: Erlangga.

15. Mamesah, D.J. 1995. SistemKeuangan Daerah. Jakarta: PT. GramediaPustakaUtama.

16. Mardiasmo. 2002. OtonomidanManajemenKeuangan Daerah. Yogyakarta: Andi.

17. Mirza, DenniSulistio. 2012. PengaruhKemiskinan, PertumbuhanEkonomi, danBelanja Modal TerhadapIndeks Pembangunan Manusia di Jawa Tengah Tahun 2006-2009. Economics Development Analysis Journal.EDAJ 1 (1).

18. Nordiawan, DeddidanAyuningtyasHertianti. 2010. AkuntansiSektorPublik. EdisiKedua. Jakarta: SalembaEmpat.

19. Rinaldi, Udin. 2012. KemandirianKeuanganDalamPelaksanaanOtonomi Daerah. JurnalEksos. Vol. 8 No. 2.

20. Saputra, Dori. 2014. AnalisisKemandiriandanEfektivitasKeuangan Daerah PadaKabupatendan Kota di Propinsi Sumatera Barat.Artikelllmiah. UniversitasNegeri Padang.

21. Sugiyono. 2014. MetodePenelitianKuantitatif, Kualitatifdan R\&D. Cetakan ke-20. Bandung: Alfabeta.

22. Sukirno, S. 2006. Ekonomi Pembangunan: Proses, Masalah, danDasarKebijakan. EdisiKedua. Jakarta: Penerbit: KencanaPrenadamedia Group.

23. Suliswanto, M.S.W. 2010.PengaruhProdukDomestikBruto (PDB) danIndeks Pembangunan Manusia (IPM) terhadapAngkaKemiskinan di Indonesia. JurnalEkonomi Pembangunan. Vol. 8 No. 2.

24. Swandewi, AnakAgungIstriAgung. 2014. Pengaruh Dana PerimbangandanKemandirianKeuangan Daerah terhadapKeserasianAnggarandanKesejahteraanMasyarakatpadaKabupaten/Kota di Provinsi Bali. EJurnalEkonomidanBisnisUniversitasUdayana 3.7.

25. Swasono, Sri Edi. 2005. Indonesia danDoktrinKesejahteraanSosial. Jakarta: Perkumpulan Prakarsa.

26. Syafii, $2009 . \quad$ Achmad. PengaruhInvestasiFisikdanInvestasi Pembangunan ManusiaTerhadapPertumbuhanEkonomiJawaTimur 1990-2004.Journal of Indonesian Applied Economics.Vol. 3 No. 1.

27. Todaro, Michael P. dan Stephen C. Smith. 2006. Pembangunan Ekonomi. AlihBahasa: MunandardanPuji. Jakarta: Erlangga.

28. Vegirawati, Titin. 2012. PengaruhAlokasiBelanjaLangsungTerhadapKualitas Pembangunan Manusia. JurnalEkonomidanInformasiAkuntansi. Vol. 2 No.1

29. Wahyudi, D., danRejekingsih, T.W. 2013.AnalisisKemiskinan di Jawa Tengah.Diponegoro Journal of Economics.Vol. 2 No.1.

30. Wahyuni I.G.A.P., $\quad$ Sukarsa, $\quad$ M., danYuliarmi, 2014.PengaruhPengeluaranPemerintahdanInvestasiTerhadapPertumbuhanEkonomidanKesenjanganPendapatan Kabupaten/Kota di Provinsi Bali.E-JurnalEkonomidanBisnisUniversitasUdayana 3.8. 
31. Widodo, A., Waridin, dan Maria, J. 2011.AnalisisPengaruhPengeluaranPemerintah di SektorPendidikandanKesehatanTerhadapPengentasanKemiskinanMelaluiPeningkatan Pembangunan Manusia di ProvinsiJawa Tengah.JurnalDinamikaEkonomi Pembangunan. Vol. 1 No. 1.

32. Undang-UndangNomorNomor 17 Tahun 2003 tentangKeuangan Negara.

33. Undang-UndangNomor 25Tahun 1999 tentangPerimbanganKeuanganantaraPemerintahPusatdan Daerah.

34. Undang-UndangNomor 1 Tahun 2004 tentangPerbendaharaan Negara.

35. Undang-UndangNomor 32 Tahun 2004 tentangPemerintahanDaerah.

36. Undang-UndangNomor 33 Tahun 2004 tentangPerimbanganKeuanganPusatdanDaerah.

37. PeraturanPemerintahNomor 58 Tahun 2005 tentangPengelolaanKeuangan Daerah.

38. PeraturanMenteriDalamnegeriNomor 13 Tahun 2006 tentangPedomanPengelolaanKeuangan Daerah.

39. PeraturanPemerintahNomor 71 Tahun 2010 tentangStandarAkuntansiPemerintahan.

40. Undang-UndangNomor 23 Tahun 2014 tentangPemerintahanDaerah.

41. http://pomphy.blogspot.com/2008/11/konsep-keuangan-daerah.html

42. http://www.kemenkeu.go.id/Berita/pemerintah-siapkan-langkah-langkah-dorong-percepatan-penyerapananggaran

\section{Attachment}

Attachment of Descriptive Analisys

Research Variable

\begin{tabular}{|c|c|c|c|c|c|}
\hline & $\begin{array}{c}\text { Local Revenue } \\
\text { Budget }\end{array}$ & $\begin{array}{c}\text { Realization of Local } \\
\text { Revenue }\end{array}$ & $\begin{array}{l}\text { Revenue of } \\
\text { Transfer }\end{array}$ & $\begin{array}{l}\text { Revenue of } \\
\text { Debt }\end{array}$ & $\begin{array}{l}\text { The budget } \\
\text { Expenditure }\end{array}$ \\
\hline 2007 & $\begin{array}{c}14.810 .965 .660 \\
00\end{array}$ & $15.821 .801 .661,30$ & $\begin{array}{c}372.661 .488 .02 \\
3,00\end{array}$ & - & $\begin{array}{c}402.337 .049 .364 \\
00\end{array}$ \\
\hline 2008 & $\begin{array}{c}\text { 13.419.093.406, } \\
00\end{array}$ & $17.460 .780 .983,52$ & $\begin{array}{c}426.600 .680 .43 \\
1,00\end{array}$ & - & $\begin{array}{c}\text { 497.316.132.764, } \\
51\end{array}$ \\
\hline 2009 & $\begin{array}{c}\text { 18.038.539.916, } \\
00\end{array}$ & $16.104 .247 .623,05$ & $\begin{array}{c}445.061 .287 .07 \\
5,00\end{array}$ & & $\begin{array}{c}\text { 496.917.080.920, } \\
00\end{array}$ \\
\hline 2010 & $\begin{array}{c}\text { 20.423.023.061, } \\
00\end{array}$ & $16.531 .437 .645,61$ & $\begin{array}{c}488.140 .888 .88 \\
2,14\end{array}$ & - & $\begin{array}{c}\text { 497.206.413.498, } \\
00\end{array}$ \\
\hline 2011 & $\begin{array}{c}\text { 19.183.910.487, } \\
00\end{array}$ & $21.551 .766 .287,81$ & $\begin{array}{c}577.771 .701 .92 \\
7,68\end{array}$ & - & $\begin{array}{c}\text { 593.392.087.117, } \\
00\end{array}$ \\
\hline 2012 & $\begin{array}{c}24.010 .110 .952, \\
00\end{array}$ & $25.894 .588 .261,47$ & $\begin{array}{c}610.184 .745 .01 \\
4,06\end{array}$ & - & $\begin{array}{c}646.091 .744 .287, \\
00\end{array}$ \\
\hline 2013 & $\begin{array}{c}\text { 34.359.972.000, } \\
00\end{array}$ & $40.096 .283 .908,94$ & $\begin{array}{c}721.603 .382 .47 \\
9,31\end{array}$ & - & $\begin{array}{c}800.900 .670 .667 \text {, } \\
00\end{array}$ \\
\hline 2014 & $\begin{array}{c}50.400 .012 .080, \\
00\end{array}$ & $60.544 .221 .463,67$ & $\begin{array}{c}785.690 .601 .33 \\
7,87\end{array}$ & - & $\begin{array}{c}\text { 887.152.936.598, } \\
00\end{array}$ \\
\hline 2015 & $\begin{array}{c}\text { 57.986.825.109, } \\
00\end{array}$ & $68.403 .420 .035,55$ & $\begin{array}{c}960.657 .598 .77 \\
2,36\end{array}$ & - & $\begin{array}{c}1.076 .441 .762 .77 \\
6,00\end{array}$ \\
\hline 2016 & $\begin{array}{c}83.286 .049 .912, \\
00\end{array}$ & $91.123 .526 .502,24$ & $\begin{array}{c}1.124 .887 .222 .1 \\
17,50\end{array}$ & - & $\begin{array}{c}1.320 .871 .298 .37 \\
0,00\end{array}$ \\
\hline Mean & $3.36 \mathrm{E}+10$ & $3.74 \mathrm{E}+10$ & $6.51 \mathrm{E}+11$ & $\mathrm{NA}$ & $7.22 \mathrm{E}+11$ \\
\hline Median & $2.22 \mathrm{E}+10$ & $2.37 \mathrm{E}+10$ & $5.94 \mathrm{E}+11$ & NA & $6.20 \mathrm{E}+11$ \\
\hline $\begin{array}{l}\text { Maxim } \\
\text { um }\end{array}$ & $8.33 \mathrm{E}+10$ & $9.11 \mathrm{E}+10$ & $1.12 \mathrm{E}+12$ & NA & $1.32 \mathrm{E}+12$ \\
\hline $\begin{array}{l}\text { Minim } \\
\text { um }\end{array}$ & $1.34 \mathrm{E}+10$ & $1.58 \mathrm{E}+10$ & $3.73 E+11$ & NA & $4.02 \mathrm{E}+11$ \\
\hline $\begin{array}{l}\text { Std. } \\
\text { Dev. }\end{array}$ & $2.31 \mathrm{E}+10$ & $2.69 \mathrm{E}+10$ & $2.47 \mathrm{E}+11$ & NA & $2.97 \mathrm{E}+11$ \\
\hline $\begin{array}{l}\text { Skewn } \\
\text { ess }\end{array}$ & 1.107154 & 0.935528 & 0.718362 & NA & 0.873012 \\
\hline
\end{tabular}




\begin{tabular}{|c|c|c|c|c|c|}
\hline $\begin{array}{c}\text { Kurtosi } \\
\mathrm{s}\end{array}$ & 2.994305 & 2.446857 & 2.367388 & NA & 2.597279 \\
\hline
\end{tabular}

\begin{tabular}{|c|c|c|c|}
\hline Realization of Expenditure & Capital Expenditure & Direct expenditure & HDI \\
\hline $364.407 .462 .557,39$ & $115.557 .711 .848,00$ & $185.901 .920 .707,20$ & 62,45 \\
\hline $452.621 .168 .584,00$ & $135.954 .041 .254,00$ & $218.156 .302 .426,00$ & 62,92 \\
\hline $489.255 .660 .055,00$ & $132.710 .582 .437,00$ & $226.990 .251 .654,00$ & 63,10 \\
\hline $480.231 .036 .478,00$ & $77.302 .748 .566,00$ & $159.354 .010 .744,00$ & 63,51 \\
\hline $559.680 .174 .950,00$ & $111.146 .742 .884,00$ & $209.075 .848 .225,00$ & 63,80 \\
\hline $600.139 .911 .637,46$ & $78.923 .645 .829,00$ & $194.948 .902 .694,00$ & 64,05 \\
\hline $741.943 .967 .305,40$ & $123.884 .355 .154,00$ & $283.024 .467 .295,00$ & 64,43 \\
\hline $841.739 .144 .164,00$ & $166.093 .334 .329,00$ & $324.972 .045 .592,00$ & 64,74 \\
\hline $997.459 .449 .170,35$ & $225.924 .896 .490,00$ & $400.381 .631 .352,00$ & 65,33 \\
\hline $1.200 .412 .475 .086,30$ & $348.422 .789 .544,00$ & $592.382 .403 .997,00$ & 65,95 \\
\hline $6.73 \mathrm{E}+11$ & $1.52 \mathrm{E}+11$ & $2.80 \mathrm{E}+11$ & 64.02800 \\
\hline $5.80 \mathrm{E}+11$ & $1.28 \mathrm{E}+11$ & $2.23 \mathrm{E}+11$ & 63.92500 \\
\hline $1.20 \mathrm{E}+12$ & $3.48 \mathrm{E}+11$ & $5.92 \mathrm{E}+11$ & 65.95000 \\
\hline $3.64 \mathrm{E}+11$ & $7.73 \mathrm{E}+10$ & $1.59 \mathrm{E}+11$ & 62.45000 \\
\hline $2.69 \mathrm{E}+11$ & $8.14 \mathrm{E}+10$ & $1.32 \mathrm{E}+11$ & 1.103407 \\
\hline 0.792010 & 1.544035 & 1.461925 & 0.304570 \\
\hline 2.454251 & 4.493208 & 4.161791 & 2.109394 \\
\hline
\end{tabular}

Independent Variable

\begin{tabular}{|c|c|c|}
\hline \multirow{2}{*}{ Year } & Independent Ratio & Effectiveness Ratio \\
\cline { 2 - 3 } & $\mathbf{X 1}$ & $\mathbf{X 2}$ \\
\hline 2007 & 4,25 & 106,82 \\
\hline 2008 & 4,09 & 130,12 \\
\hline 2009 & 3,62 & 89,28 \\
\hline 2010 & 3,39 & 80,95 \\
\hline 2011 & 3,73 & 112,34 \\
\hline 2012 & 4,24 & 107,85 \\
\hline 2013 & 5,56 & 116,69 \\
\hline 2014 & 7,71 & 120,13 \\
\hline 2015 & 7,12 & 117,96 \\
\hline 2016 & 8,10 & 109,41 \\
\hline Mean & 1.593 .481 & 4.684 .104 \\
\hline Median & 1.445 .666 & 4.708 .331 \\
\hline Maximum & 2.091 .948 & 4.868 .449 \\
\hline Minimum & 1.219 .830 & 4.393 .771 \\
\hline Std. Dev. & 0.331565 & 0.141824 \\
\hline
\end{tabular}




\begin{tabular}{|c|c|c|}
\hline Skewness & 0.485543 & -0.946898 \\
\hline Kurtosis & 1.608 .648 & 3.002 .117 \\
\hline
\end{tabular}

Intervening Variable

\begin{tabular}{|c|c|c|c|c|}
\hline \multirow[t]{2}{*}{ Year } & Expenditure Confirmity Ratio & Budget Absorption Ratio & \multirow[b]{3}{*}{$\begin{array}{c}\text { Expenditure } \\
\text { Conformity } \\
\text { Ratio }\end{array}$} & \multirow[b]{3}{*}{$\begin{array}{c}\text { Budget } \\
\text { Absorption } \\
\text { Ratio } \\
\end{array}$} \\
\hline & $\mathbf{X 3}$ & $\mathbf{X 4}$ & & \\
\hline 2007 & 62,16 & 90,57 & & \\
\hline 2008 & 62,32 & 91,01 & $\mathrm{X3}$ & $\mathrm{X} 4$ \\
\hline 2009 & 58,47 & 98,46 & & \\
\hline 2010 & 48,51 & 96,59 & & \\
\hline 2011 & 53,16 & 94,32 & & \\
\hline 2012 & 40,48 & 92,89 & & \\
\hline 2013 & 43,77 & 92,64 & & \\
\hline 2014 & 51,11 & 94,88 & & \\
\hline 2015 & 56,43 & 92,66 & & \\
\hline 2016 & 58,82 & 90,88 & & \\
\hline Mean & 3.970 .679 & 4.537 .513 & & \\
\hline Median & 4.003 .139 & 4.530 .178 & & \\
\hline Maximum & 4.132 .275 & 4.589 .632 & & \\
\hline Minimum & 3.700 .914 & 4.506 .153 & & \\
\hline Std. Dev. & 0.146964 & 0.027425 & & \\
\hline Skewness & -0.623318 & 0.616527 & & \\
\hline Kurtosis & 2.184 .568 & 2.334 .811 & & \\
\hline
\end{tabular}

Dependent Variable

\begin{tabular}{|c|c|}
\hline \multirow{2}{*}{ Year } & HDI \\
\cline { 2 - 2 } & $\mathbf{Y}$ \\
\hline 2007 & 62,45 \\
\hline 2008 & 62,92 \\
\hline 2009 & 63,10 \\
\hline 2010 & 63,51 \\
\hline 2011 & 63,80 \\
\hline 2012 & 64,05 \\
\hline 2013 & 64,43 \\
\hline 2014 & 64,74 \\
\hline 2015 & 65,33 \\
\hline 2016 & 65,95 \\
\hline Mean & 4.159 .187 \\
\hline Median & 4.157 .709 \\
\hline Maximum & 4.188 .897 \\
\hline Minimum & 4.134 .366 \\
\hline Std. Dev. & 0.017194 \\
\hline
\end{tabular}




\begin{tabular}{|c|c|}
\hline Skewness & 0.279716 \\
\hline Kurtosis & 2.094 .394 \\
\hline
\end{tabular}

\section{Attachment. Regression Analysis}

Equation1 :The Effect X1 and X2 on X3

Dependent Variable: X3

Method: Generalized Linear Model (Quadratic Hill Climbing)

Date: 08/29/18 Time: 05:07

Sample: 20072016

Included observations: 10

Family: Normal

Link: Identity

Dispersion computed using Pearson Chi-Square

Convergence achieved after 1 iteration

Coefficient covariance computed using observed Hessian

\begin{tabular}{|c|c|c|c|c|}
\hline Variable & Coefficient & Std. Error & z-Statistic & Prob. \\
\hline $\mathrm{X} 1$ & 2.188446 & 0.987685 & 2.215732 & 0.0267 \\
\hline $\mathrm{X} 2$ & 0.013765 & 0.173992 & 0.079110 & 0.9369 \\
\hline $\mathrm{C}$ & 71.57516 & 21.04659 & 3.400796 & 0.0007 \\
\hline Mean dependent var & 95.91730 & \multicolumn{2}{|c|}{ S.D. dependent var } & 25.82611 \\
\hline Sum squared resid & 3469.020 & \multicolumn{2}{|c|}{ Log likelihood } & -43.71797 \\
\hline Akaike info criterion & 9.343594 & \multicolumn{2}{|c|}{ Schwarz criterion } & 9.434370 \\
\hline Hannan-Quinn criter. & 9.244014 & \multicolumn{2}{|c|}{ Deviance } & 3469.020 \\
\hline Deviance statistic & 495.5742 & \multicolumn{2}{|c|}{ Restr. deviance } & 6002.891 \\
\hline LR statistic & 5.113001 & \multicolumn{2}{|c|}{ Prob(LR statistic) } & 0.077576 \\
\hline Pearson SSR & 3469.020 & \multirow{2}{*}{\multicolumn{2}{|c|}{ Pearson statistic }} & 495.5742 \\
\hline Dispersion & 495.5742 & & & \\
\hline
\end{tabular}

\section{Equation 2 :The Effect $\mathrm{X} 1$ and $\mathrm{X} 2$ on $\mathrm{X} 4$}

Dependent Variable: X4

Method: Generalized Linear Model (Quadratic Hill Climbing)

Date: 08/29/18 Time: 05:08

Sample: 20072016

Included observations: 10

Family: Normal

Link: Identity

Dispersion computed using Pearson Chi-Square

Convergence achieved after 1 iteration

Coefficient covariance computed using observed Hessian

\begin{tabular}{|c|r|r|r|r|}
\hline & Coefficient & Std. Error & Z-Statistic & Prob. \\
\hline \hline Variable & & & & \\
\hline $\mathrm{X} 1$ & 2.763606 & 1.323430 & 2.088215 & 0.0368 \\
\hline $\mathrm{X} 2$ & -0.251348 & 0.233137 & -1.078117 & 0.2810 \\
\hline $\mathrm{C}$ & 60.08211 & 28.20097 & 2.130498 & 0.0331 \\
\hline
\end{tabular}




\begin{tabular}{|l|r|l|r|}
\hline Mean dependent var & 61.89530 & S.D. dependent var & 34.31902 \\
\hline Sum squared resid & 6228.326 & Log likelihood & -46.64415 \\
\hline Akaike info criterion & 9.928830 & Schwarz criterion & 10.01961 \\
\hline Hannan-Quinn criter. & 9.829249 & Deviance & 6228.326 \\
\hline Deviance statistic & 889.7609 & Restr. deviance & 10600.15 \\
\hline LR statistic & 4.913487 & Prob(LR statistic) & 0.085714 \\
\hline Pearson SSR & 6228.326 & Pearson statistic & 889.7609 \\
\hline Dispersion & 889.7609 & & \\
\hline \hline
\end{tabular}

Equation 3 :The Effect $X 3$ and $X 4$ on $Y$

\begin{tabular}{|c|c|c|c|c|}
\hline \multicolumn{3}{|l|}{ Dependent Variable: Y } & & \\
\hline \multicolumn{5}{|c|}{ Method: Generalized Linear Model (Quadratic Hill Climbing) } \\
\hline \multicolumn{3}{|c|}{ Date: 08/29/18 Time: 05:10 } & & \\
\hline \multicolumn{3}{|c|}{ Sample: 20072016} & & \\
\hline \multicolumn{3}{|c|}{ Included observations: 10} & & \\
\hline \multicolumn{2}{|c|}{ Family: Normal } & & & \\
\hline \multicolumn{2}{|l|}{ Link: Identity } & & & \\
\hline \multicolumn{4}{|c|}{ Dispersion computed using Pearson Chi-Square } & \\
\hline \multicolumn{4}{|c|}{ Convergence achieved after 1 iteration } & \\
\hline \multicolumn{5}{|c|}{ Coefficient covariance computed using observed Hessian } \\
\hline Variable & Coefficient & Std. Error & Z-Statistic & Prob. \\
\hline $\mathrm{X} 3$ & 0.035291 & 0.017337 & 2.035604 & 0.0418 \\
\hline $\mathrm{X} 4$ & 0.028880 & 0.013047 & 2.213585 & 0.0269 \\
\hline $\mathrm{C}$ & 59.29215 & 1.945923 & 30.46995 & 0.0000 \\
\hline Mean dependent var & 64.46470 & \multicolumn{2}{|c|}{ S.D. dependent var } & 1.755487 \\
\hline Sum squared resid & 12.56697 & \multicolumn{2}{|c|}{ Log likelihood } & -15.61519 \\
\hline Akaike info criterion & 3.723039 & \multicolumn{2}{|c|}{ Schwarz criterion } & 3.813814 \\
\hline Hannan-Quinn criter. & 3.623458 & \multicolumn{2}{|l|}{ Deviance } & 12.56697 \\
\hline Deviance statistic & 1.795281 & \multicolumn{2}{|c|}{ Restr. deviance } & 27.73563 \\
\hline LR statistic & 8.449185 & \multicolumn{2}{|c|}{ Prob(LR statistic) } & 0.014631 \\
\hline Pearson SSR & 12.56697 & \multicolumn{2}{|c|}{ Pearson statistic } & 1.795281 \\
\hline Dispersion & 1.795281 & & & \\
\hline
\end{tabular}

